\section{“ARTERIAL EMBOLECTOMY"*}

BY

\section{GEOFFREY JEFFERSON, M.S.LOND., F.R.C.S.ENG} HONORARY SURGEON, SALFORD ROYAL HOSPITAL, ETC.

From time to time it falls to the lot of most of us to advise on the treatment of an embolus lodged at some high level in a peripheral artery (subclavian, axillary, brachial, iliac, femoral, popliteal, and even at the aortic bifurcation).

The ideal treatment for arterial embolism, which is a grave emergency calling for intervention as early as, or even earlier than, that in respect of a perforated viscus, is immediate arteriotomy, removal of clot, and suture of the vessel. This procedure, when successful, restores the circulation, and gangrene is averted. Experience drawn from other types' of gangrene, such as the senile, arteriosclerotic, and diabetic types, is quite useless; the rarer embolic form is a distinct and separate entity, which can often be prevented by immediate and decisive surgery.

Several tentative attempts to this end were made during the first decade of this century (Lejar, 1902 ; Moynihan, 1903 ; Sampson Handley, 1907 ; Proust and Lecène, 1908 ; Carrel and Leriche, 1909), but without success. At the turn of the first decade Carrel's experimenial work on the surgery of the blood vessels demonstrated that, given a careful technique, very fine silk suture material, and the use of anticoagulants in the wound, vessels could be incised and closed again without thrombosis at the suture line. His work, and that of others in the same field, paved the way for successiul embolectomy, and it was but a matter of oppoitunity before some more venturesome surgeon would be able to claim a success. This fell to the lot of Labey, who in 1911 removed an embolus arrested for six hours in the femoral artery. Since that date numerous cases have been put on record, some successful, others not, and at the present day embolectomy can be regarded as a standardized and useful addition to surgery. This is in no small degree due to the Swedish surgical school, led by Einar Key, whos masterly papers in 1922 and 1927 laid the foundations on which all else has been built up. In recording my own successful case in 1925 I was able to collect from tho literature seventy-three cases, of which twenty-eight had done well. Since then the literature has grown prodigiousiy, and important analyses have been made by Petitpierre (118 cases, 1928), H. E. Pearse (296 cases, 1933), and Max Danzis (detailed analysis of all cases reported, 1922-32). The time has arrived when general compilations have done their very useful work in driving home the lessons of embolectomy: future general studies are likely to be concerned with embolisms of individual vessels rather than discussions of the whole subject comprehensively.

I do not intend, therefore, to make a new survey of the whole field as $\mathrm{I}$ did in 1925, for Danzis's paper, in particular, has brought the whole subject sufficiently up to date. A better purpose will be served by an account of my personal experiences of embolectomy. The 1925 case was the first successful one in this country, and I have now five new cases to report. Two other British successes have, in the meanwhile, been obtained individually by A. G. Banks and G. E. Larks, and others will be recorded to-day.

* Read in opening a discussion in the Section of Surgery at the Annual Meeting of the British Medical Association, Bournemouth, 1934.

\section{Personal Cases}

The six cases which follow record embolisms in the axillo-brachial junction, brachial, common iliac, and femoral arteries, four of which have been diagnosed and immediately referred to me by my colleague Dr. G. J. Langley.

\section{CASE I}

This case has already been reported, being my original success of 1925 . It concerned a man of 42 years of age, operated upon at Salford Royal Hospital on April 23rd, 1925, for radical cure of an umbilical hernia. Twenty-six hours after operation he complained of pins-and-needles in the left arm, with increasing numbness and paralysis of all muscles below the elbow. The pain rapidly became intense. The arm was blanched, cold, and pulseless below the anterior fold ot the axilla. Palpation of the subclavian artery above the clavicle gave free pulsation there, and pulsation was felt again, but not so distinctly, in the axilla. Under local anaesthesia the junction of the axillary and brachial arteries was cut down upon two and a half hours later, a $2 \mathrm{~cm}$. long incision was made in its wall, and a clot removed. The artery was sewn up with fine paraffined silk, with immediate return of colour and muscular power, and cessation of pain. The pat:ent made a perfect recovery, and is well nine years later.

Comment.-This was a completely successful case, pulsation returning at once, and all symptoms disappearing immediately. Banks and Larks have published very similar and equally successful cases since; both were axillo-brachial, both occurred in hospital, and both were post-operative.

\section{CASE II}

W. P., male, aged 49 (date, $10 / 12 / 31$ ), after a period of ill-health, developed an acute and semi-gangrenous ischiorectal abscess. He was seen by Dr. Langley, who found a very high blood sugar, and the patient was removed to a nursing home in order to have the abscess opened by my colleague Mr. Garnett Wright. In the early hours of the morning, about 3 o'clock, the patient was awakened from sleep by severe pain in the left arm, which he attributed to a draught. When seen by Dr. Langley later in the morning the patient complained of exceedingly severe pain, and on examination the arm was found to be pale, cold, and pulseless-indeed, no pulse could be felt in the axilla. The projected

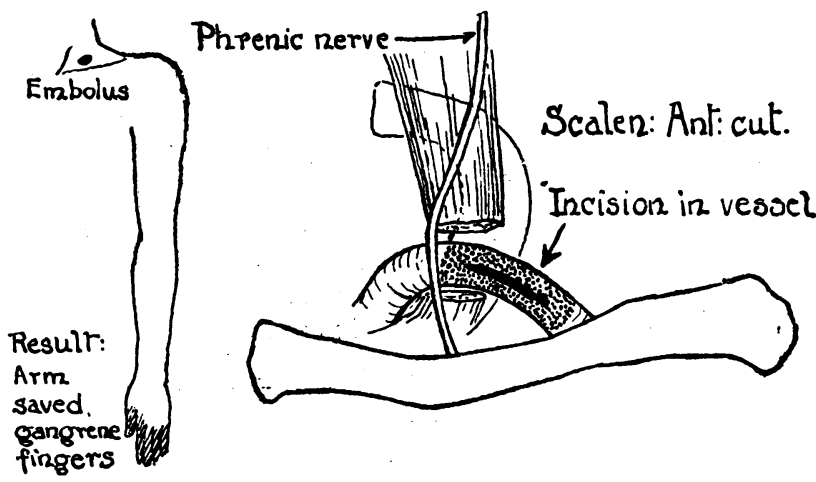

cpening of the ischio-rectal abscess was therefore postponed until the arm could be dealt with. I saw the patient with Mr. Wright and Dr. Langley at noon. All the classical signs of acute arterial obstruction were present-a cold, pallid, marbled limb, with wrist-drop, and absence of all finger movements. Flexion and extension of the elbow could be feebly carried out. No pulsation could be detected in any artery of the limb, but above the clavicle, on deep inward pressure, a muffled beat could be felt. The embolus was evidently lodged in the subclavian artery in its first or second part, and operation was decided upon. At 6 p.m., fifteen hours after lodgement, the left subclavian artery was exposed through a transverse incision under omnopon, scopolamine, and gas-oxygen anaesthesia. The clavicular head of tho sterno-mastoid muscles was divided, the phrenic nerve retracted inwards, and the scalenus anticus cut through at its 
attachment to the first rib. This gave excellent exposure of the artery, in which an embolus could be both seen and felt. The artery was isolated, and two fine controlling bands of rubber tissue threaded round it above and below the clot. A short incision was made in the vessel and the main clot milked out in one piece. A glass suction tube was inserted, and more clot fragments obtained by judicious release of first one and then the other ligature. The arterial incision was closed with interrupted vaselined fine silk, a difficult task in this situation. The immediate result was good, the pulse returning feebly at the wrist, but next morning it could not be felt, though the pain had largely gone and movement had returned in some measure to the hand. During the ensuing days this power disappeared again, and in the long run a considerable amount of tissue had to be removed from the hand, including all the fingers.

Comment.-A point of considerable interest is the origin of the clot in cases such as this with a surgical lesion. The thrombus might come from the auricle, as presumably occurs in the cases of auricular fibrillation. Bull showed that most arterial emboli are cardiac in origin, and only come from great vessels when there is gross disease in the intima. But paradoxical embolism has to be considered, for a patent foramen ovale is present in a number of cases. It seems unlikely that an aseptic clot will arise from a septic area, such as was present in this case, but it is not impossible, for I have seen the median cephalic vein thrombose slowly along its whole length in the arm of a man with a septic wound of the dorsum of the hand. No sign of infection and no tenderness, certainly no cellulitis, developed over the vein, so that a propagating clot, which is in large part at least aseptic, may arise from a septic area. Case II cannot be regarded as a complete failure ; for without operation an amputation above the elbow-joint would certainly have been required. The patient's arm now is vastly superior to any artificial limb that was ever invented.

\section{CASE III}

S. T. J., male, aged 42 (date, 3/2/32), was admitted to the Salford Royal Hospital under the care of Dr. G. J. Langley suffering from auricular fibrillation. He was treated

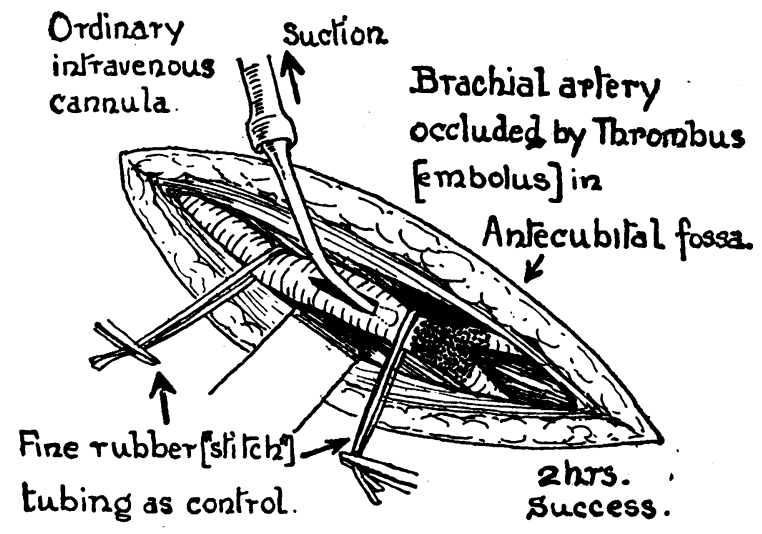

with massive doses of digitalis, and on $3 / 2 / 32$, at 9 p.m., complained of sudden violent pain and weakness in the left hand. On examination no pulses could be felt at the wrist or in the hand; pulsation in the brachial artery could be felt in the lower third of the arm, but ceased in the antecubital fossa. Diagnosis was made of embolus at the bifurcation of the brachial artery, and two and a half hours later, under local anaesthesia, this vessel and the upper ends of the radial and ulnar arteries were exposed. A clot was removed by incision into the brachial artery, and recovery ensued, but with feeble pulses at the wrist. There was a slight degree of ischaemic contracture of the hand and the wrist in this case, a point of considerable pathological interest. This patient was lost sight of on leaving hospital, but he died of syncope whilst on holiday six months later.
Comment.-This case furnished an interesting example of the damage which an embolus will do as compared with arterial ligature at the same level. In the antecubital fossa the brachial can be tied with impunity, yet an embolus, by blocking all collaterals, causes a very severe circulatory disturbance, and the hand may well be lost. In this case collaterals were undoubtedly freed, but I doubt if the full blood stream was restored in the brachial artery past the point of embolism and arteriotomy. At the present day I should not open the brachial artery as soon as it was exposed, as I did in actual fact. It is better to conserve the main trunk and to milk the clot,

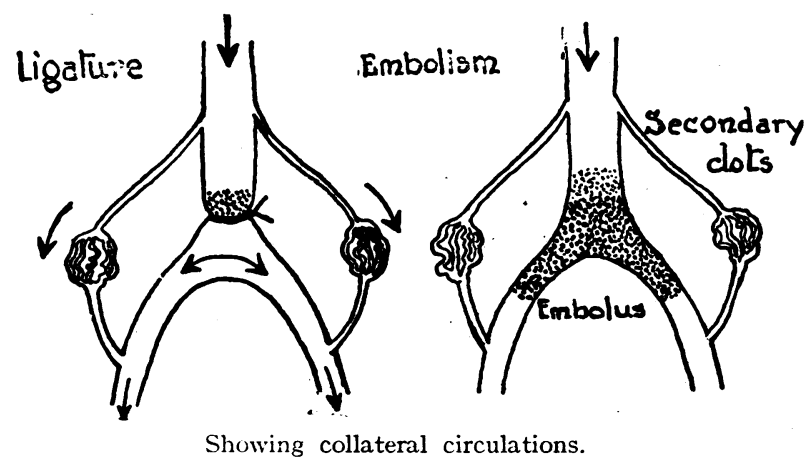

under rubber ligature control, into a vessel of lesser importance, as I did in Case $\mathrm{v}$, and as Nyström, Schmorell, and Westerborn have so brilliantly done in their cases of aortic embolism. I shall refer to this point in technique again later.

\section{CASE IV}

Mrs, Y., aged 66 (date, 20/10/33), a doctor's wife, was admitted to a nursing home under the care of Dr. Langley with high-speed auricular fibrillation. At 6 a.m. she complained of severe pain in the left lower limb. Seen three and a half hours later the limb was cold to the touch, pallid, and powerless, with no pulse in the femoral artery at Poupart's ligament. The right femoral pulse was strong. It was probable, therefore, that the embolus was lodged at the bifurcation of the common iliac artery, and operation was advised and accepted. Under spinal anaesthesia the left common iliac artery was exposed, five hours after embolism, extraperitoneally by cutting through the flat muscles of the abdomen. A large embolus could be felt riding the bifurcation of this vessel, with short limbs projecting into the external and internal iliac arteries. The common iliac was lightly controlled with improvised forceps covered with rubber tubing, and the other two branches controlled by tension bands of thin rubber (" stitch") tubing. An incision $1.5 \mathrm{~cm}$. long was made into the external iliac artery, below the embolus, a suction nozzle was introduced into the empty artery, and an attempt made to break up the clot. This failed until a blunt pituitary spoon was used, when, in a moment or two, the whole $\mathrm{Y}$-shaped embolus came away. Momentary release of the controlling forceps verified the absence of any further clot. The artery was sutured with interrupted sutures of arterial silk, and proved to be watertight after three sutures had been placed. Recovery was complete and perfect. In spite of a small cerebral embolism two months later, the effects of which passed away completely, she is alive and well ten months later, and walls well.

Comment.-This was a completely successful case. It was in many respects a severe test of the operation. There was complete arterial block, and a high-level amputation would have had to be done if the arteriotomy had failed. Amputation in a case with severe cardiac disease is a more difficult obstacle to surmount than an embolectomy, not only immediately, but later on. To a patient with heart disease the loss of a lower limb is a most serious thing, because of the added exertion on movement. 
CASE V

Mrs. E., aged 52 (date, 28/2/34), was admitted to Salford Royal Hospital by Dr. G. J. Langley, who had seen her in consultation with Dr. Andrew Clarke. Some years ago she had an attack of aphasia, but, apart from recent auricular fibrillation on an old-standing mitral stenosis, had been reasonably well and active. At 10.30 a.m. on 28/2/34 she felt sudden severe pain in the left leg, and signs of embolism at the level of the profunda femoris -were classically present. The clot, which made a palpable and visible swelling in the artery, was easily removed under spinal anaesthesia through a $1.5 \mathrm{~cm}$. incision under full control. The immediate effect appeared to be good, though the absence of feeling in the legs prevented discriminating observation. The vessel below the

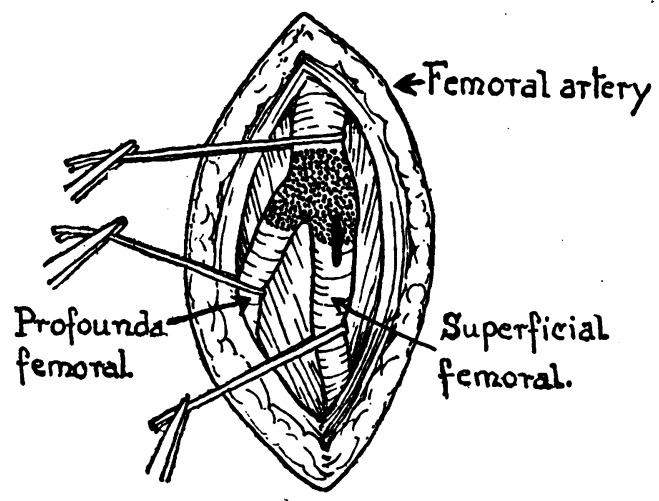

arteriotomy wound had been noted to be unduly small before the skin was sutured, but this is not uncommon, as vessels contract primarily when stripped. Next day she still complained of pain in the foot, though higher up the circulation had greatly improved. During the next few days the pain became so intense that $1 / 2$ to 1 grain of morphine a day did not control it. Finally, fifteen days after embolism, the patient consented at last to amputation above the knee. A second embolism was found blocking the posterior tibial and an abnormal peroneal artery. Two days later she suddenly developed signs of embolism in the femoral artery of the other side, but just as operation was about to be undertaken all signs and symptoms disappeared, and colour and pulsation returned to the leg. Evidently the embolus had side-tracked into the profunda artery. On 21/3/34 the patient died, with signs suggesting an aortic embolus.

Comment.-This case was a failure, and exemplifies the disadvantages of spinal anaesthesia. There is no escaping its use in iliac and aortic embolism, but in the limbs infiltration anaesthesia is far preferable, as it allows of immediate observations of the effect of the operation on pain and mobility in the limb. Had it been used here we should at once have been sure that something else was wrong, and either an arteriogram would have been made or the popliteal artery exposed. A small embolus was found in the amputated leg in the posterior tibial artery. I believe these distal embolisms to be most serious. Arteriograms in cases of thrombo-angiitis obliterans show how apparently trivial deficiencies will lead to distal gangrene. To be sure, the conditions are not entirely parallel, for there is no general arterial disease involving small vessels in the embolic cases. The pain experienced by this patient was pitiable in its severity, and would have been an object-lesson to those few who think that embolisms are best left alone.

\section{CASE VI}

Mrs. M., aged 48 (date, $13 / 4 / 34$ ), a patient of Dr. Sissons of Lymm, had long suffered from thyrotoxicosis and auricular fibrillation. Three weeks prior to the present attack she had had a sudden attack of pain in the right leg, which became pale, dead, and powerless. At the end of two or three hours, after having kept it warm, and after vigorous massage, it recovered spontaneously. At 2 a.m. on April 13th, 1934, she experienced the same pain in the right arm. Thinking that the symptoms would pass off as they had done in the leg, she did not send for Dr. Sissons until six hours later, when he at once diagnosed a brachial embolism, but she appeared to be dying. As the day wore on she slowly improved generally, and as her physical condition improved so the pain in the arm became more distresing. At 9 p.m., nineteen hours after embolism, a clot was removed under local anaesthesia from the upper end of the right brachial artery at the level of the superior profunda branch. The clot was extracted without incident, but although there was a free flow of blood from the upper end further clot was felt and seen lower down. A second incision was made in the lower third of the arm, and a further small incision made, and the long clot milked out. The pulses still did not return to the wrist, and by this time the patient had developed increasing pain in the abdomen, suggesting an embolism of the mesenteric vessels. The attempt to restore the circulation in the arm was therefore abandoned. Next morning the arm was warm as low as the wrist, and pulsation was present in the antecubital fossa. Pain in the abdomen was by this time very severe, and blood-stained diarrhoea set in, confirming the suspicion of intestinal embolism. She died the next day, forty-eight hours after the embolism.

Comment.-This case would, I think, have been a failure had the patient survived. Of course, her mesenteric embolism made recovery impossible. But this was an interesting case, showing how rapidly secondary thrombus formation occurs in the distal part of the vessel ; for, nineteen hours after occlusion, the brachial artery, and no doubt the more distal vessels too, contained long rods of clot. Her medical attendant had wished for an embolectomy at once, but her immediate state of collapse prevented an intervention; no blame therefore attaches to him.

To these cases of proven embolism must be added one in which the left iliac bifurcation was exposed under a mistaken diagnosis. The condition found was uncommon, and the indication for exploration and the decision to operate were, I think, not unsound.

\section{CASE VII}

J. C., male, aged 33 (date, 14/12/29), consulted his medical attendant, Dr. Young of Kersal, for pain over the heart. Dr. Young made a diagnosis of ulcerative endocarditis and aortic disease, and sent him to bed. At the end of a week his dyspnoea improved, and he got up on his own responsibility. At 8 p.m. he experienced a sudden pain in the left leg, and at 8.30 p.m. was seen in consultation by Dr. Langley, who found the leg to be pulseless and cold. The very proper diagnosis of acute arterial occlusion was made, no doubt embolic, and he was sent into a nursing home. At 11.15 p.m., three and a quarter hours after the presumed embolism, I explored the left common iliac artery. No pulse was present in the left common femoral, and as the right leg was quite unaffected the occlusion should not have been aortic, but somewhere in the iliac arteries, probably at the bifurcation of the common iliac. Under general anaesthesia an oblique incision was made above the inguinal ligament, and the muscles divided. By blunt dissection the bifurcation of the iliac arteries was brought into view, and free pulsation found there. Following the external iliac down, there was seen to be a dark haemorrhage, $4 \mathrm{~cm}$. long, occupying the anterior half of the circumference of the vessel. This had caused so much swelling of the arterial wall that no blood could get past it. This haematoma was incised, but was found to be in the middle as well as the adventitial coat of the artery. The pathology of the condition was clearly the earliest possible stage of a dissectrng aneurysm, due to softening of a segment of the artery by lodgement of an infected particle (such as is a commonplace of ulcerative endocarditis) in a vas vascrum. It seemed that nothing useful could be done, though, to be sure, after the relief of tension in the wall by incision feeble pulsation was detected in the femoral but not in the popliteal artery nor at the ankle. The wound was closed, and next day the leg was slightly warmer but far from normal. The patient died on the third day, the combination of unfavourable circumstances being too much for him. 


\section{Clinical Picture}

The clinical history of the patients in the foregoing series is remarkably constant, and there has been no great difficulty in arriving at a diagnosis (except, of course, in Case viI). This is important, because it is the general practitioner who sees these cases first, and everything depends on the promptitude with which he makes his decision and acts upon it, for he has at best four hours, and at most ten hours, in which to have an arteriotomy performed.

Most of the subjects are sufferers from heart disease with some form of myocardial damage, and auricular stasis with unsuspected clot formation. They stand the relatively benign operation very well. A smaller number occur as post-operative disasters in hospital, and this group undoubtedly has the best chance of survival, for reasons which will occur to everyone.

The picture can best be summed up as consisting of pain, pallor, and paralysis, with absent pulsation in the vessels of the limb. The mechanism of the production of pain of the intensity experienced by these patients is difficult to explain. For the moment we may rest without an explanation, because it is an unescapable fact that diminution in the blood supply to a limb invariably produces pain. Raynaud's disease, intermittent claudication, and even the vaso-constriction produced by cold are all obvious examples. Muscular paralysis is a less familiar result of the same thing, and may puzzle the clinician who has had no experience of embolism. In a case only partially relieved, contracture of the muscle can occur through death of some of its fibres (as happened in Case III). Cutaneous anaesthesia is also found in embolism, sometimes in a known anatomical distribution, and more often with a less recognizable pattern. Nerve endings require an efficient circulation for their proper function; the anaesthesia is therefore more apt to have a vascular distribution than a purely nervous one, though anaemia of nerve trunks as well as of nerve endings must be taken into account in explaining the distribution of anaesthesias and paralyses.

\section{Localization of the Embolus}

There have been no mistakes in the localization of the embolus in my own cases. Difficulties are only likely to arise in cases with multiple embolisms (such as Case v), or when the clot slips from its first point of lodgement to be arrested at a lower level. Emboli usually lodge at bifurcations, because the arterial lumen narrows suddenly at the points where large branches are given off. Clots are usually of some size, and on the whole it is more probable that a large rather than a small vessel has been obstructed, otherwise the symptoms would not have arisen.

The ictus is not always as dramatic as in the cases described above, but develops from relatively small beginnings, with paraesthesias and considerable, but not intense, pain associated with pale blotching of the limb. In these cases the embolus has not completely blocked the vessel, and rides the spur at an important bifurcation, allowing a diminished blood stream to flow past. Some such cases make a sudden and spontaneous recovery (as in the first embolism in Case vi), and this occurrence has puzzled some observers, who have invoked spasm rather than clot obstruction as the explanation. In such cases it is justifiable to assume that the clot, after riding for a time and impeding the flow in the main nutrient artery of the limb, suddenly disappears into the less important of the two branches. Thus with a clot insecurely lodged at the common iliac or common femoral bifurcations, the thrombus might on occasion pass into the internal iliac or profunda femoris arteries, where it would do little harm. When the embolus is large, and at once firmly and definitively plugs the vessel, the pain and all other symptoms and signs are instantaneous, and steadily worsen as the hours pass.

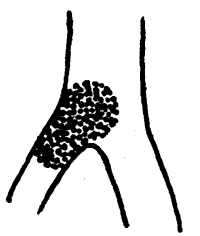

$\Lambda$

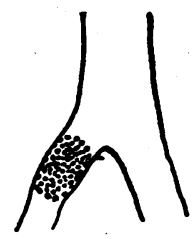

B
A, Clot, chiefly in large branch, partly occluding the main blood supply. B, clot hammered into branch releasing circulation to distal limb.

If there is great difficulty in localizing the site of embolism, help may be obtained from an arteriogram, an idea already put into successful practice by Pearse (see also Abbeloos). Failure to find the obstruction at the expected point is remedied by injection of thorotrast or uroselectan into the artery and a radiograph taken as the solution is injected. I have so far no experience of the method, but it is sound practice.

\section{Treatment}

Treatment need not detain us long, for most of the points are inherent to what has gone before. The artery should be exposed with as little delay as is compatible with making the diagnosis and the removal of the patient to a suitable place. The time factor is important, because secondary clot formation beyond the embolus sets in after a few hours, but not immediately. I am no believer in the view advanced by some that it is not until secondary thrombosis occurs that the signs become definite. The vessel distal to the point of occlusion is empty during the first few hours, and ready to accommodate the blood stream as soon as the obstruction is removed.

I believe that the failures that sometimes follow early operation are due to there being more than one embolus, rather than because of coagulation at the point of incision into the artery. The use of local anaesthesia as opposed to spinal is important, as the patient's subjective sensations are most helpful in deciding whether immediate success has been achieved. I have indicated briefly: what should be done if the result is not at once satisfactory. There is one point remaining which requires discussion, and it arises out of the treatment of aortic embolism. I have seen three such cases, but in none of them was operation possible for various reasons (the patient's state too desperate or the embolus lodged too long). Nyström and Key both advise the massaging or " milking" of the clot from the aortic bifurcation down into the common femorals under direct vision (the femoral arteries having both been exposed and controlled by rubber bands to prevent the broken clot lodging at some less convenient site). In a case of Hildebrandt's, reported by Schmorell, the clot suddenly and unexpectedly disappeared into the internal iliac artery as it was being massaged down to the exposed femoral artery, with immediate return of function to the limb. I have referred to such a side-tracking as the explanation of the sudden recoveries that may undoubtedly occur in embolisms before such time as the clot becomes adherent to the intima, but it seems to me that a practical hint might be taken from these happy chances, and that when it does not happen of itself we should try to compel it. The first step would therefore be the exposure and temporary control of the main vessel above and below the embolus. 
in the ordinary, followed by a deliberate attempt to massage the clot into the subsidiary, branch. If this failed the operation would be proceeded with on normal lines and the artery opened. It is likely that the alternative method will not always succeed ; in the late cases it certainly cannot, but it is worth a trial. I must be very explicit as to my exact meaning, for I think that no greater disservice could be done a patient than a haphazard breaking up of the clot by finger pressure, allowing the resulting smaller emboli to go free in the circulation. We should then have multiplicity and small size of emboli, two factors which I have come to regard as most unfavourable. It is far easier to remove an embolus from the femoral artery than from the posterior tibial, so that if the clot cannot be persuaded into the profunda femoris, for example, it must at once be removed by arteriotomy without for one instant relaxing the clamps or temporary ligatures which are preventing the escape of the clot.

\section{BIBLIOGRAPHY}

Abbeloos, J. B.: “ Localisation des Emboles Artériels par l'Artériographie," Journ. de Chir. et Ann. Soc. Belge de Chir., March, 1933.

Banks, A. G.: "A Case of Brachial Embolectomy," British Medical Journal, 1932, i, 56.

Bull, P.: "What can more than 6,000 Post-mortem Examinations Teach Us about Emboli and Embolic Gangrene of the Extremities?" Acta Chir. Scand., 1922, liv, 315

Carrel, A., and Guthrie, C. "C.: "Reversal of the Circulation in a Limb," Ann. of Surg., 1906, xliii, 203.

Danzis, Max: "Arterial Embolectomy," ibid., 1933, xcviii, 249.

Gordon-Watson, C.: "A Case of Limbolectomy," British Medical Journal 1926, i, 1032; " Post-operative Entbolism of the Femoral Artery," Clin. Journ., 1913

Handley, Sampson: "An Operation for Embolus," British Medical Journal, 1907, i, 712 .

Jefferson, G.: "A Successfu' Case of Embolectomy," ibid., 1925, ii, 985.

Key, E.: “Uber Embolektonie als Behandlungsmethode bei Embolischen Funktionsstorungen der Extremitaten," Acta Chir. Scand., 1922, liv, 339 ; Zentralbl. f. Chir., 1927, liv, 2190.

Larks G. "E.: "A Case of Axillo-brachial Embolectomy." British Medical Journal, 1934, i, 616 .

Moynihan, Lord: "An Operation for Embolus," ibid., 1907, ii, 826.

Neuhof, H.: "Embolectomy with Partial Arterial Occlusion for Embolism of the Extremities," Ann. of Surg., 1932, xcti, 44.

Nyström, G.: "Zur Prognose und Methodik der Embolektomie," Acta Chir. Scand., 1926, lx, 229.

Pearse, H. E.: "Embolectomy for Arterial Embolism of the Extremities," Ann. of Surg., 1933, xcviii, 17 .

Petitpierre, M.: "Embolie der Extremitaten Arterien," Schweiz. med. Woch., 1928, xxviii, 700 .

Proust, H.: "Embolie de l'Artère Femorale: Artériotomie," Bull. et Mém. Soc. de Chir., 1911, N.S., xxxvii, 1094

Schmorell, H.: "Erfolgreiche Operation bei reitenden Embolús auf der Aortenbifurkation," Zentralbl. f. Chir., 1933, lx, 1509.

Westerborn, A.: "Zwei mit Erfolg operierte Falle von wiederholten der Aartenbifurkation," Zentralbl. f. Chir., 1933, lx, 1509.

According to P. Esch (Zentralbl. f. Gynäk., October 27th, 1934 , p. 2530), many cases previously described and accepted as osteomalacia have in reality been examples of generalized osteodystrophia fibrosa (von Recklinghausen's Disease). $\mathrm{He}$ gives details of two cases in which this mistake had been made; in both, radiological examinations of the skeleton, and in one necropsy, showed the characteristic signs of generalized bony fibrosis, with " brown cysts" in certain bones. The patient who died had adenomata in three parathyroid glands. Distinction between osteomalacia and von Recklinghausen's disease is important: in, one of Esch's cases of the latter, removal of one and irradiation of the other ovary had been unnecessarily done for ${ }^{\circ}$ a supposed osteomalacia, and on the other hand successful treatment by parathyroidectomy is possible in some cases of osteodystrophia fibrosa. In diagnosis radiology is the most important measure. The pelvic deformity in von Recklinghausen's disease, if symmetrical, causes marked lateral flattening: the pubis projects forward beakwise, the ascending rami of the pubis are nearly parallel, and the inlet takes the shape of the heart on a playing card. Accepting the view that Paget's disease and von Recklinghausen's disease are different varieties of osteodystrophia fibrosa, Esch points out finally that the non-generalized form of the former may begin in the lumbar vertebrae or the pelvis, so that early cases may come first to the gynaecologist.

\section{RUPTURED SPLEEN AFTER TRIFLING MISHAPS}

A RECORD OF TWO CASES

BY

HAROLD DODD, M.CH., F.R.C.S.

The cases described below are of interest, owing to the apparently trivial domestic incidents which excited a serious abdominal lesion-that is, a ruptured spleen. These accidents initiated slight but progressive symptoms and physical signs, necessitating splenectomy. Fortunately, judging from the literature, the condition appears to be an uncommon one.

\section{Case I}

Frank P., forty-eight hours before admission to hospital, fell against a gas-oven door and bumped his left lower ribs. This accident gave rise to no immediate effects beyond temporary pain. A few hours later he vomited several times and had diarrhoea, but he stated that he saw no blood or slime in the stools. In twelve hours he had developed general abdominal pain, slightly worse in the upper region. After the initial looseness there was no further action of the bowels, so that when he was admitted to hospital he had been constipated for about forty hours.

Winen I saw him first his general condition was good; he was complaining of abdominal discomfort, but not of definite

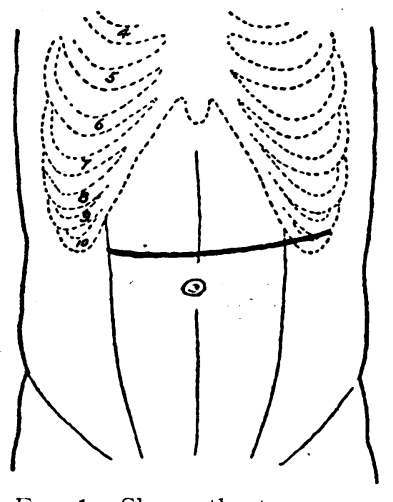

Fig. 1.-Shows the transverse incision.

pain. His temperature was $100^{\circ}$, pulse 110 , and respirations 20. The abdominal reflexes were normal, and there was no hyperaesthesia. In the right iliac fossa was a visible fulness with a vaguely defined soft swelling; he was tender on pressure here-that is, over the appendix and caecum. $\mathrm{He}$ was slightly sensitive over the lower left ribs external to and below the nipple, the point of contact with the oven door, but this was apparently outside the abdominal area. The diagnosis of an atypical acute appendix or " something odd" in the abdomen was made. The possibility of the fall having caused an intra-abdominal traumatic lesion, such as a ruptured spleen, was considered and rejected on account of the seeming slightness of the mishap, and also of the clinical picture.

Immediate operation was advised, and was performed under a percaine spinal anaesthetic. Owing to the element of doubt in the diagnosis, the abdomen was opened through a right paramedian incision centred at the umbilicus. Blood shone through the peritoneum and poured out freely when it was opened. This recalled the abandoned suspicion of a ruptured spleen. The peritoneum was rapidly closed and a transverse incision made through the upper left abdomen (Fig. 1). Through this approach the spleen was easily delivered from its surroundings of blood clot; its vessels were ligatured, and it was quickly removed. On dividing the peritoneum the second time the patient collapsed, became pulseless, and seemed as if he were dead. Intracardiac adrenaline and intravenous saline were administered, and after these his pulse faintly returned. The entire procedure was brief, and while the abdomen was being closed the patient was turned into the Trendelenburg position, extra blankets being tucked around him, and electric stoves placed near by. In an hour's time 